
R. SCHIEßL $\mathrm{L}^{1, \infty, *}$
U. MAAS $^{2}$
A. HOFFMANN ${ }^{3}$
J. WOLFRUM ${ }^{3}$
C. SCHULZ 3,4

\title{
Method for absolute OH-concentration measurements in premixed flames by LIF and numerical simulations
}

\footnotetext{
${ }^{1}$ Yale Center for Laser Diagnostics, Yale University, P.O. Box 208286, New Haven, CT 06520-8286, USA

${ }^{2}$ Institut für Technische Thermodynamik, Universität Karlsruhe, Kaiserstrasse 12, 76128 Karlsruhe, Germany

${ }^{3}$ PCI, Universität Heidelberg, INF 253, 69120 Heidelberg, Germany

${ }^{4}$ IVG, Universität Duisburg-Essen, 47057 Duisburg, Germany
}

\section{Received: 1 June 2004 \\ Published online: 29 July 2004 • @ Springer-Verlag 2004}

ABSTRACT We present and apply a methodology for the singleshot measurement of absolute concentrations of the $\mathrm{OH}$-radical in a turbulent, premixed natural gas/air flame. The method is based on a combination of detailed numerical simulations of the turbulent flame and an experimental approach using planar laser-induced fluorescence (LIF). The numerical simulation is used to predict LIF intensities. It shows the existence of a sharp correlation between the LIF signal after excitation of the $A-X(3,0) P_{2}(8)$ transition near $248.45 \mathrm{~nm}$ and $\mathrm{OH}$ concentrations for a wide range of conditions, including stationary and instationary laminar flames of different strain rates, with different models to treat molecular transport and different degrees of heat loss. This correlation allows the transformation of measured $\mathrm{OH}-\mathrm{LIF}$ intensity images into absolute $\mathrm{OH}$ concentration maps.

PACS 82.33.Vx; 82.20.Wt; 42.62.Fi

\section{Nomenclature}

\begin{tabular}{lll}
$\varphi$ & - & equivalence ratio \\
$T$ & $\mathrm{~K}$ & temperature \\
$T_{\mathrm{u}}$ & $\mathrm{K}$ & temperature of unburned fuel/air mixture \\
$p$ & $\mathrm{~Pa}$ & pressure \\
$x_{i}$ & - & mole fraction of species $i$ \\
$c_{i}$ & moles $/ \mathrm{m}^{3}$ number density (concentration) of species $i$ \\
{$[\mathrm{OH}]$} & $\mathrm{moles} / \mathrm{m}^{3}$ OH number density (concentration) \\
$\psi$ & $\mathrm{Pa}, \mathrm{K}$ & state vector, $\psi \equiv\left(p, T, x_{1}, \ldots, x_{n S}\right)$ \\
\hline$n_{\mathrm{S}}$ & - & number of chemical species \\
$a$ & $\mathrm{~s}-1$ & strain rate \\
$v$ & $\mathrm{~m} / \mathrm{s}$ & speed \\
$z$ & $\mathrm{~m}$ & spatial coordinate \\
$\omega$ & $\mathrm{s}$ & mixing rate \\
$J_{\mathrm{rot}}$ & - & rotational quantum number \\
$Z$ & - & partition function \\
$\mathrm{LIF}$ & - & LIF-signal \\
$\mathrm{LIF}$ & - & normalized LIF-signal \\
$I$ & $\mathrm{~J} /\left(\mathrm{m}^{2} \mathrm{~s}\right)$ & light intensity \\
$h$ & $\mathrm{~J} \mathrm{~s}$ & Planck's constant $6.62 \times 10^{-34} \mathrm{~J} \mathrm{~s}$ \\
$c_{\mathrm{vac}}$ & $\mathrm{m} / \mathrm{s}$ & speed of light in vacuo $2.9989 \times 10^{8} \mathrm{~m} / \mathrm{s}$ \\
& &
\end{tabular}

Fax: 1-203/432-6775, E-mail: robert.schiess1@yale.edu

${ }^{*}$ Current address: Dr. Ing. Robert Schieß1, Center for Laser Diagnostics, Yale University, P.O. Box 208286, New Haven, CT 06520-8286, USA
1

\section{Introduction}

Absolute concentration measurements of chemical species in flames are crucial for the development of quantitative combustion models, or for a detailed investigation of a given combustion device. It is often desired to gain multidimensional information, either to obtain a more complete image of the combustion in a spatially inhomogeneous system (like the combustion chamber of an engine or a gas turbine), or to infer explicit information about the local gradients of a quantity, e.g., in a flame front.

In this work, we present and apply a method for measuring concentrations of the hydroxyl radical $(\mathrm{OH})$ using laserinduced fluorescence (LIF). The approach is based on detailed numerical modelling of both the thermochemical states in a methane/air combustion system and the LIF-spectroscopy of $\mathrm{OH}$.

The problem in performing absolute concentration measurements with LIF is generally that the relationship between the observed quantity (mostly, the intensity of the emitted fluorescence) and the desired quantity (e.g., the concentration of the fluorescence emitter) is often rather complex. The signal is almost always influenced by more than only one species, via effects like collisional deactivation of the laser-excited state (quenching) or pressure broadening of absorption lines. Also, it often displays a strong temperature-dependence via the thermal population of the laser-coupled ground state. Formally expressed, the measured signal $S$ is a function $f$ of all components of the state vector $\psi \equiv\left(p, T, x_{i}\right), S=f(\psi)$, not only of one single variable. This means that the observable signal does not provide sufficient information to deduce the value of any of these variables, as the equation $S-f\left(p, T, x_{i}\right)=0$ is underdetermined.

We show, however, that the situation becomes much better if we consider that the different components of the state vector are not independent from each other. Correlations exist that link concentrations of different species, and species concentrations and temperature together; these correlations provide additional information that can be combined with the results from the measurements. By properly exploiting these correlations, much of the "classical" problems in LIF measurements (like quenching or changes in thermal population of the lasercoupled ground state) can be mitigated or even completely eliminated. 
In this work, we perform a wide range of numerical simulations in order to collect all the possible state vectors that can be realized in a premixed, turbulent methane/air flame. These simulations include laminar one-dimensional flames with different strain rates, stoichiometries, and specific enthalpies. Additionally, state vectors resulting from homogeneous reactor calculations are considered, so as to account for instationary effects and for mixing of air into the flame gases. From this large set of accessible state vectors, OH-LIF signals are calculated. We show that a quite sharp and unique correlation between the LIF signal and $\mathrm{OH}$ number density exists; in fact, the correlation is sharp, unique and universal enough to be used for the determination of absolute $\mathrm{OH}$ number densities from $\mathrm{OH}-\mathrm{LIF}$ measurements in a wide range of premixed methane/air-flames, without requiring further information or assumptions about temperature fields or the quenching environment. We present an application of the method to absolute measurements of $\mathrm{OH}$ concentrations in a turbulent, premixed natural-gas/air flame.

\section{2}

\section{Methodology}

The method for measuring absolute values of $\mathrm{OH}$ is based on measuring LIF signal intensities LIF, and transforming them into $\mathrm{OH}$ number densities $[\mathrm{OH}]$ by using computed correlations between LIF and $[\mathrm{OH}]$.

The OH-LIF signal LIF measured in an experiment is given by

$$
\mathrm{LIF} \sim I_{\mathrm{laser}}[\mathrm{OH}] f_{\mathrm{B}}(T) \sigma_{\mathrm{eff}} A_{21} /\left(A_{21}+Q_{21}\left(p, T, x_{i}\right)+P\right),
$$

with the incident laser intensity $I_{\text {laser }}$ and the instantaneous temperature $T . f_{\mathrm{B}}(T)$ is the fractional part of molecules in the laser-coupled ground state (Boltzmann fraction), $\sigma_{\text {eff }}$ is the effective absorption cross-section (i.e., the absorption crosssection where the finite spectral width of the laser and the absorption line are regarded), $A_{21}$ is the Einstein coefficient for spontaneous emission and $Q_{21}$ and $P$ are quenching and predissociation rates, respectively. $A_{21}$ and $P$ are specific to the excitation/detection strategy and independent of the state vector $\left(p, T, x_{i}\right)$. Commonly, $\sigma_{\text {eff }}$ and the quenching rate are, due to their strong dependence on collisions a rather complex function of $p, T$ and $x_{i}$. In our approach, a predissociative upper level was excited with the laser, so that $A_{21}, Q_{21} \ll P$. The rate for the spontaneous emission $A_{21}$ in the $(3,0)$ band is of the order $1.6 \times 10^{4} \mathrm{~s}^{-1}$ and for quenching of the order of approximately $10^{9} \mathrm{~s}^{-1}$ depending on the properties of the surroundings like temperature and quenching partners. The order of predissociation is about $1 \times 10^{10} \mathrm{~s}^{-1}$. Compared to this effect of collisional quenching at the considered conditions (a pressure of $1 \mathrm{bar}$ ) is therefore negligible. Note, the approach presented here is not limited to predissociative LIF. In "conventional" LIF schemes, however, the influence of the state vector on $Q$ and $T$ must be incorporated as well.

We express the measured OH LIF in units of a normalized signal LIF $_{\text {standard }}$ that is obtained in a standard calibration flame (see below, [1]), with known laser-intensity, temperature, pressure and species mole fractions. We use the symbol $\mathrm{LIF}_{\mathrm{OH}}$ for this normalized signal (a dimensionless quantity), in order to distinguish it from the actual LIF signal (in units of intensity counts of the employed detector):

$\mathrm{LIF}_{\mathrm{OH}}=\mathrm{LIF} / \mathrm{LIF}_{\text {standard }}$,

where LIF $_{\text {standard }}$ is the LIF signal measured in a standard flame with the same experimental setup (i.e., the same pump laser intensity, same characteristics of the employed light collection system and of the detector) as the actual measurement. $\mathrm{LIF}_{\mathrm{OH}}$ is then a function of $[\mathrm{OH}]$ and $T$ only, $\mathrm{LIF}_{\mathrm{OH}}=$ $f([\mathrm{OH}], T)$, and does not depend on a particular experimental situation. This allows us to get rid of purely experimental parameters (laser intensity, collection efficiency of the employed detection line) and constant spectroscopic terms (namely, $A_{21} / P$ and $\sigma_{\text {eff }}$, and also photo-bleaching effects in the case of constant pump laser irradiance). Note that in the case of varying pressures, the pressure-dependent variation of the line-shapes must be included. In that case, $f$ is a more complex function of the state vector.

In our case with predissociative LIF we can make the simplification that the only temperature influence on the measured $\mathrm{OH}-\mathrm{LIF}$ signal is through the Boltzmann fraction. We assume that vibrational energy transfer to neighboring states (where the assumption of fast predissociation rates is not correct) is negligible. In fact the emission from the $A-X(2,1)$ transition falls partially into the detected wavelength range and contributes approx. $30 \%$ to the over-all signal. If this signal contribution is varied due to collisional quenching by $30 \%$ an over-all error of $7 \%$ would result that we consider negligible.

In a turbulent flame, a large number of different scenarios (combinations of $T$ and $[\mathrm{OH}]$ ) can occur as a consequence of the complicated interaction between chemical reactions, turbulent flow and molecular transport. It is our strategy to search for correlations between $\mathrm{LIF}_{\mathrm{OH}}$ and $[\mathrm{OH}]$ that occur in the flame; if they exist, we strive to determine $[\mathrm{OH}]$ from the measured value $\mathrm{LIF}_{\mathrm{OH}}$. Due to changes in Boltzmannfraction, $\mathrm{LIF}_{\mathrm{OH}}$ is a function $f([\mathrm{OH}], T)$; if this function is known, $[\mathrm{OH}]$ can be determined by solving the nonlinear equation

$\mathrm{LIF}_{\mathrm{OH}}-f([\mathrm{OH}], T)=0$.

The function $f[\mathrm{OH}], T$ can be determined by spectroscopical modelling. Equation (3) is one equation in two unknowns $([\mathrm{OH}]$ and $T$ ). By considering results from numerical simulations, an additional relationship $R([\mathrm{OH}], T)$ can be added to (3), and a unique solution may become possible. The equation system then reads

$$
\begin{aligned}
& \mathrm{LIF}_{\mathrm{OH}}-f([\mathrm{OH}], T)=0 \\
& R([\mathrm{OH}], T)=0
\end{aligned}
$$

This is a system of two equations for the two unknowns [OH] and $T$. The function $f([\mathrm{OH}], T)$ depends on the experimental configuration (the LIF-excitation and -detection scheme, as well as the experimental setup). $R([\mathrm{OH}], T)$ is independent of any experimental issues, but reflects correlations that are intrinsic to the thermochemical and physical properties of the combustion system under investigation. If, by favorable selection of the experimental configuration, functions $f([\mathrm{OH}], T)$ 
and $R([\mathrm{OH}], T)$ can be established so that they have appropriate mathematical properties, a unique determination of $[\mathrm{OH}]$ from a measured value $\mathrm{LIF}_{\mathrm{OH}}$ is possible. It is even possible (provided that, for the given combustion system, an experimental configuration can be found so that $f([\mathrm{OH}], T)$ and $R([\mathrm{OH}], T)$ have the right mathematical properties) that both $[\mathrm{OH}]$ and $T$ can be derived from the system; however, we focus exclusively on $[\mathrm{OH}]$ in this paper.

\section{Experimental procedure \\ 3.1 The turbulent flame}

The investigated object is a highly turbulent, premixed natural gas/air flame that is stabilized on a bluff-body burner. The equivalence ratio of $\varphi=0.95 \pm 0.01$ was controlled by mass-flow controllers. The burner consists of a steel tube with an outer diameter of $50 \mathrm{~mm}$ and an inner diameter of $45 \mathrm{~mm}$ with a concentric bluff-body (dia. $35 \mathrm{~mm}$ ). A natural gas/air mixture flows $\left(55 \mathrm{~m}^{3} / \mathrm{min}\right)$ through the crevice between tube and bluff-body. The Reynolds number of the cold flow is about 15000 . The burner is enveloped by a co-flow of filtered air (exit diameter $200 \mathrm{~mm}$ ) to guarantee dust-free flame gases and ambient air, and to reduce the shear forces between the flame and the surroundings. In the flame a strong recirculating vortex transports hot burned gases continuously towards the region close to the burner exit and therefore stabilizes the combustion process. The shape of the flame is statistically axisymmetric, and the visible flame cone is about $50 \mathrm{~cm}$ long. A considerable entrainment of air into the burned gases can be observed. Especially, with increasing height above the burner this effect increases. The overall flame structure has been characterized in previous work by point-wise Raman measurements that were performed at many different radial and axial locations in the flame [2]. For automated measurements at arbitrary points in the flame the burner is mounted on a precision $x y$-positioning stage.

\subsection{OH-LIF measurements}

Measurements of $[\mathrm{OH}]$ with planar LIF The technique of planar laser-induced fluorescence is a measurement technique which is common for the detection of two-dimensional species concentrations [3-5]. However, to quantify the measured concentration distributions, additional temperature information is needed to correct for the $T$-dependence. These temperature fields must be measured simultaneously to allow the measurement of instantaneous species concentrations which is often a problem of limited optical access to the measured plane [6]. An alternative is to excite ground-state levels where the $T$-dependence of $f_{\mathrm{B}}(T)$ (or the over-all $T$-dependence of the LIF signal [7]) is minimized in the expected temperature range. In the present case we measured $\mathrm{OH}-\mathrm{LIF}$ intensities in the described turbulent burner and we apply the approach presented above of quantifying LIF signals by combining measurement techniques with concentration-temperature-correlation-information obtained from numerical simulations.

A narrowband $\mathrm{KrF}$ excimer laser (Lambda Physik, EMG 150 TMSC, $160 \mathrm{~mJ}$ per pulse, $0.5 \mathrm{~cm}^{-1}$ line width) is tuned

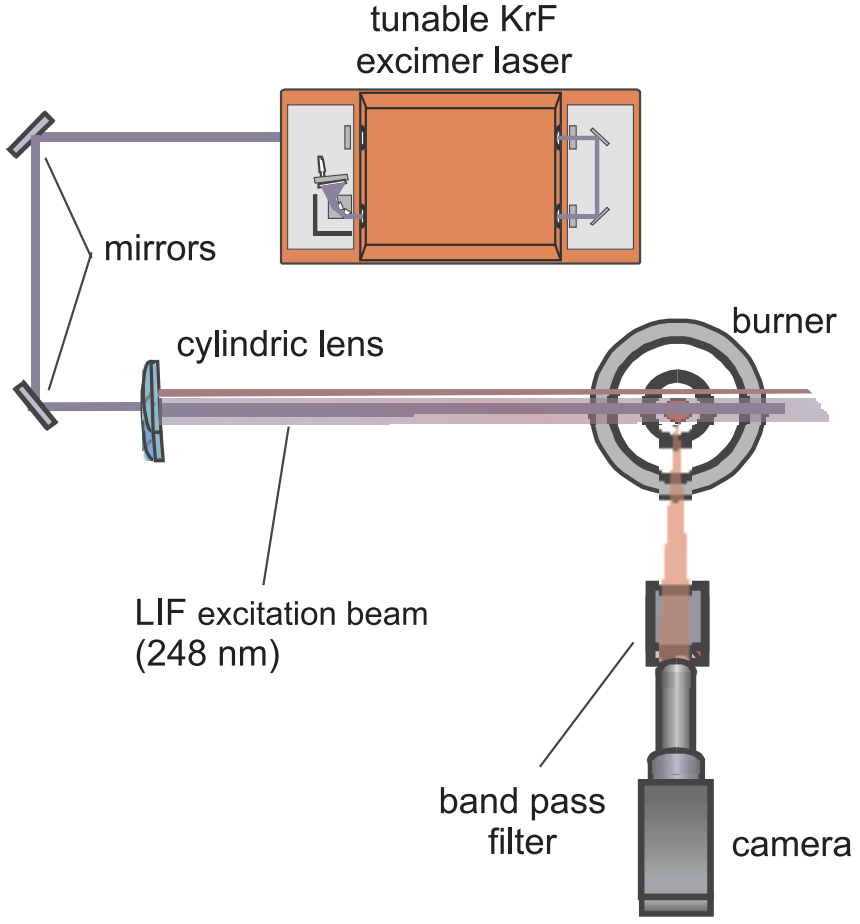

FIGURE 1 Experimental setup

to excite $\mathrm{OH}$ in the $A-X(3,0) P_{2}(8)$ transition at $248.45 \mathrm{~nm}$. The beam is focused cylindrically to form a $300 \mu \mathrm{m}$ thick light sheet that crosses the observed volume (Fig. 1). The spectral irradiance of the laser beam was $85 \mathrm{MW} / \mathrm{cm}^{2} / \mathrm{cm}^{-1}$. A reflection band pass filter (Laseroptik, $295 \pm 10 \mathrm{~nm}$ ) separates the OH-LIF signal emitted from the $A-X(3,2)$ band from interference. Signal is then imaged by a intensified CCDcamera (LaVision, StreakStar) with a UV lens (Halle, UV lens, $f=300 \mathrm{~mm}, f_{\#}=5.0$ ). As outlined above, the measured signal LIF is normalized to the OH-LIF signal LIF $_{\text {standard }}$ measured in a McKenna burner at well defined conditions [1]; thus, the normalized LIF signal $\mathrm{LIF}_{\mathrm{OH}}$ is obtained.

Photochemical effects have been reported for the excitation of $\mathrm{OH}$ in the $A-X(3,0)$ band with a $\mathrm{KrF}$ excimer laser with high spectral irradiances. Photodissociation of $\mathrm{H}_{2} \mathrm{O}$ can double the measured $\mathrm{OH}-\mathrm{LIF}$ signals as reported by Nguyen et al. [8]. In their work, significant excess signals were observed at 300-400 ns after the laser pulse. In order to minimize the impact of photodissociation, we detect the signal within $200 \mathrm{~ns}$ after the laser pulse. For laser irradiances above $20 \mathrm{MW} / \mathrm{cm}^{2} / \mathrm{cm}^{-1}$, photobleaching effects of the laser-coupled ground states have been reported, too. They reduce the signal by $\sim 25 \%$ for irradiances $>$ $100 \mathrm{MW} / \mathrm{cm}^{2} / \mathrm{cm}^{-1}$ [8]. But as photobleaching affects the calibration measurement to the same extent, it can be neglected here, as long as the laser irradiance properties are constant throughout the observed area (i.e., no significant absorption of pump laser light occurs).

\section{$4 \quad$ Numerical procedure}

The basic idea of our approach is to collect all scenarios (combinations of temperature and species concentrations) that the system can achieve, and to calculate the 
$\mathrm{OH}-\mathrm{LIF}$ signal $\mathrm{LIF}_{\mathrm{OH}}$ for these scenarios. Then, we seek for correlations between $\mathrm{LIF}_{\mathrm{OH}}$ and $[\mathrm{OH}]$, and use these to determine the desired quantity $[\mathrm{OH}]$ from the directly observable quantity $\mathrm{LIF}_{\mathrm{OH}}$. For this purpose, a set of numerical calculations was performed.

To model the thermochemical behavior of the flame, including the effects of molecular transport and detailed chemistry, one-dimensional calculations of a premixed flame embedded in a counterflow configuration were performed (Fig. 2, frame a, see Maas and Warnatz [9], and Stahl and Warnatz [10] for a detailed description of the underlying equations and the numerical procedure). The chemical source term needed for the calculations was based on a detailed methane/air mechanism, involving 295 elementary reactions of 34 species [10]. For the one-dimensional flame calculations, different transport models were regarded: a model with Lewis-number $L e=1$, one with $L e \neq 1$, but equal species diffusivities, and a model with detailed treatment of molecular transport for heat and each individual species, including the Dufour- and Soret-effect.

The feeds on both sides contained unburned methane/air mixture at nominally the same temperature $\left(T_{0}=300 \mathrm{~K}\right)$, pressure ( $p=1 \mathrm{bar}$ ) and chemical composition (fuel/air equivalence ratio $\varphi_{0}=0.95$ ) like in the experiment. To determine the sensitivity of the results with respect to these input parameters, calculations were performed where $\varphi_{0}$ and $T_{0}$ were varied in a range around the nominal values.
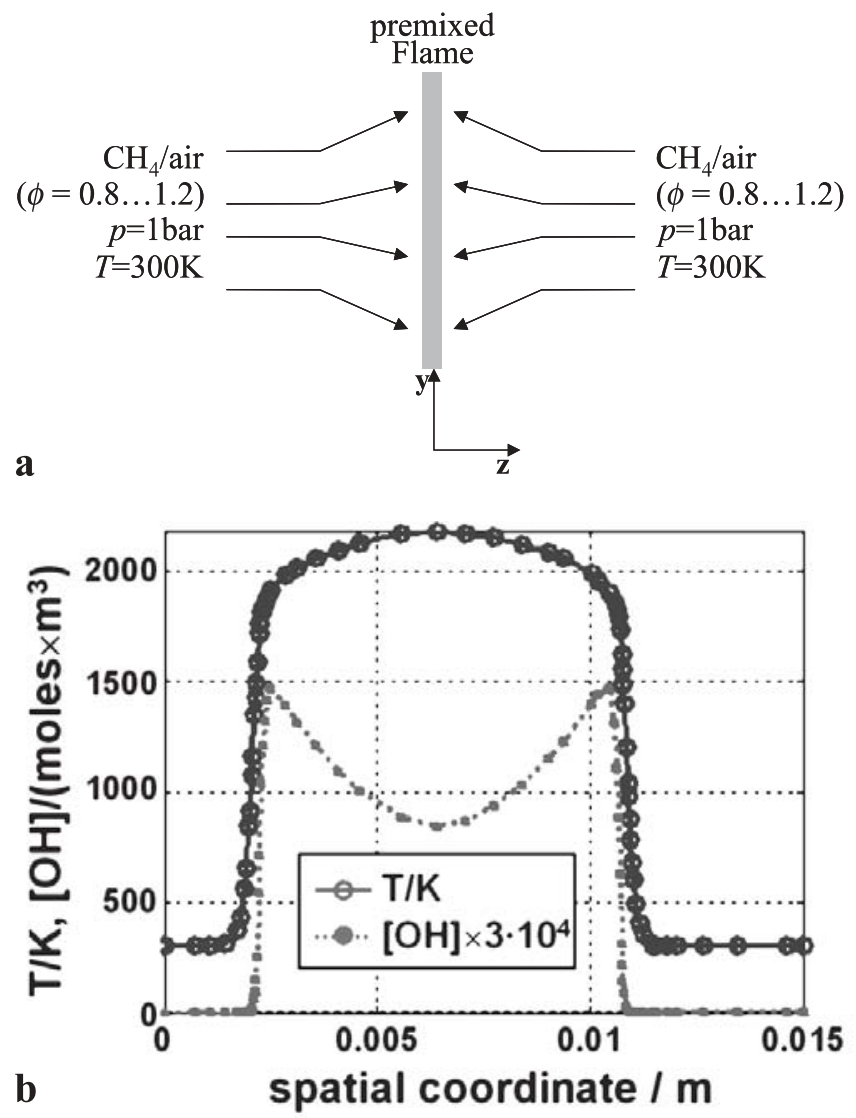

FIGURE 2 a Schematic of the 1D-premixed counter-flow flame configuration. b simulated spatial temperature- and $[\mathrm{OH}]$-profiles of one of the sample flames
The flow rates of the feeds were also varied over a wide range, in order to model the different strain rates that the flame experiences in the highly-turbulent flow of our experiment. The computational domain had a width of $1.5 \mathrm{~cm}$, with fixed boundary conditions for temperature and mixture composition at both sides. The calculations were performed until a stationary solution was achieved. Both stationary and instationary flames were taken into account; the instationary flames were obtained by simulating the transition from the stationary state at one flow rate to the stationary state at another flow rate. Spatial profiles of $T$ and $[\mathrm{OH}]$ (stationary solution) are depicted in Fig. 2, frame b, to exemplify the simulation results.

Furthermore, studies with a perfectly stirred reactor model were performed: a homogeneous reactor is initially filled with unburned gas, and burned gases are added according to a given mixing rate $\omega$. The mixing rate specifies the time scale at which mass from the fresh gas stream is added to the reactor, and can be viewed as equivalent to the strain rate $a$ of flame simulations. Simultaneously to the mixing process, chemical reactions occur in the reactor. Due to this competition of mixing and chemical reactions, the system does not reach chemical equilibrium, but attains a stationary state somewhat away from equilibrium.

The reactions in the mixing reactor are described by the same detailed chemical mechanism for $\mathrm{CH}_{4}$ /air combustion as used for the flame calculations above. The reactor model was also employed to study the mixing of air with burned gases; this corresponds to the entrainment of ambient air into the flame, an effect that certainly occurs at some locations in our flame.

For all results from the flame and homogeneous mixing reactor simulations, $\mathrm{LIF}_{\mathrm{OH}}$ was calculated as a function of the state vector using (2) for each point in space and time. For numerical evaluations of $\mathrm{LIF}_{\mathrm{OH}}$ the following (4) was used:

$$
\begin{aligned}
\mathrm{LIF}_{\mathrm{OH}}= & \alpha[\mathrm{OH}] f_{\mathrm{B}}(T)=\alpha[\mathrm{OH}]\left(2 J_{\mathrm{rot}}+1\right) \\
& / Z(T) \exp \left(-E_{0} /\left(k_{\mathrm{B}} T\right)\right) \\
f_{\mathrm{B}}(T)= & \left(2 J_{\text {rot }}+1\right) / Z(T) \exp \left(-E_{0} /\left(k_{\mathrm{B}} T\right)\right) \\
\alpha= & \left(I / I_{\text {standard }}\right)\left(K / K_{\text {standard }}\right) /\left([\mathrm{OH}]_{\text {standard }} f_{\mathrm{B}}\left(T_{\text {standard }}\right)\right) .
\end{aligned}
$$

$I_{\text {standard }}, K_{\text {standard }}, T_{\text {standard }},[\mathrm{OH}]_{\text {standard }}$ are the values of laser intensity, detection efficiency, temperature and $\mathrm{OH}$ concentration in the standard flame, respectively. The Boltzmann factor $f_{\mathrm{B}}(T)$ accounts for the population of the excited ground state. In the formula, $\left(2 J_{\text {rot }}+1\right)$ represents the degeneracy of the excited ground state, $Z(T)$ the partition function, $E_{0}$ the ground state energy, $k_{\mathrm{B}}$ Boltzmann's constant and $T$ the temperature. The values used for the description of the ground state are listed below. The partition function $Z(T)$ is approximated by a Taylor series up to the third term. The following data and formulae complete the numerical expression for $\mathrm{LIF}_{\mathrm{OH}}$ : The rotational quantum number of the excited ground state is $J_{\text {rot }}=8$. The energy $E_{0}$ of the ground state is $7.3 \times 10^{-21} \mathrm{~J}$. The approximated formula for the description of the partition function $Z(T)$ of this level is

$Z(T)=17.051+0.10233 T / \mathrm{K}+2.369 \times 10^{-5} T^{2} / \mathrm{K}^{2}$. 


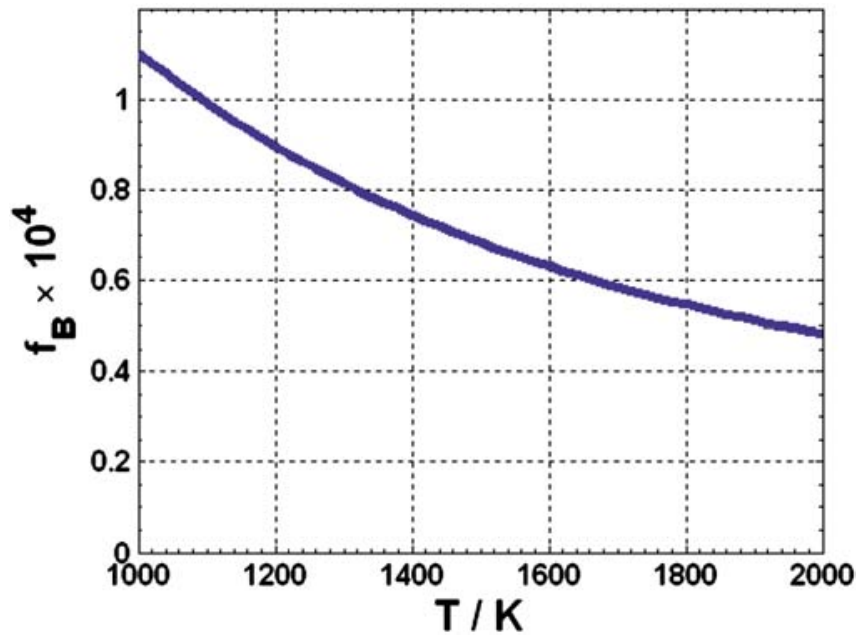

FIGURE 3 The $T$-dependent Boltzmann factor $f_{\mathrm{B}}(T)$ in the temperature range between $1000 \mathrm{~K}$ and $2000 \mathrm{~K}$

The influence of the temperature dependent Boltzmann factor within the combustion relevant temperature range $(\mathrm{OH}$ is only present in the range between 1200 and $2000 \mathrm{~K}$ ) is shown in Fig. 3. The influence of this factor can vary significantly when switching to a different excitation scheme.

Further values given for the quantification of the $\mathrm{OH}-$ LIF signal are the concentration $[\mathrm{OH}]_{\text {standard }}$ measured in a standard McKenna burner at a given position of $15 \mathrm{~mm}$ above the burner matrix at the local flame temperature $T_{\text {standard }}[1]$

$[\mathrm{OH}]_{\text {standard }}=0.00498 \mathrm{~mol} / \mathrm{m}^{3}$

$T_{\text {standard }}=1879 \mathrm{~K}$.

5

5.1

\section{Results}

\section{Correlations between LIF signals and $\mathrm{OH}$} concentrations

Frame a) of Fig. 4 shows the computed correlation between $[\mathrm{OH}]$ and $\mathrm{LIF}_{\mathrm{OH}}$, as obtained from the premixed 1D-flame calculations. The diagram is a scatter plot showing all results from a large number of flamelet calculations, performed for the following conditions: The strain rate $a$ varied in the range $10 \mathrm{~s}^{-1}$ to $5000 \mathrm{~s}^{-1}$, initial temperatures $T_{\mathrm{u}}$ varied in the range $280 \mathrm{~K}-350 \mathrm{~K}$, equivalence ratios varied between $\varphi=0.85$ and $\varphi=1.1$, and different mixing models $(L e=1$ and detailed transport model). The straight line shows the relationship $[\mathrm{OH}]=0.00541 \mathrm{~mol} / \mathrm{m}^{3} \times \mathrm{LIF}_{\mathrm{OH}}$. It is seen that the correlation for these 1D counterflow-flames is quite universal with respect to variation of the transport model, $T_{\mathrm{u}}, \varphi, a$ and also quite sharp, although not quite unique for $\mathrm{LIF}_{\mathrm{OH}}>3.5$. The relative uncertainty in $[\mathrm{OH}]$ due to this non-uniqueness is, however, small (below $\pm 15 \%$ throughout the whole range of accessible LIF signals). Moreover, the correlation is almost linear (although this property is not required for our method).
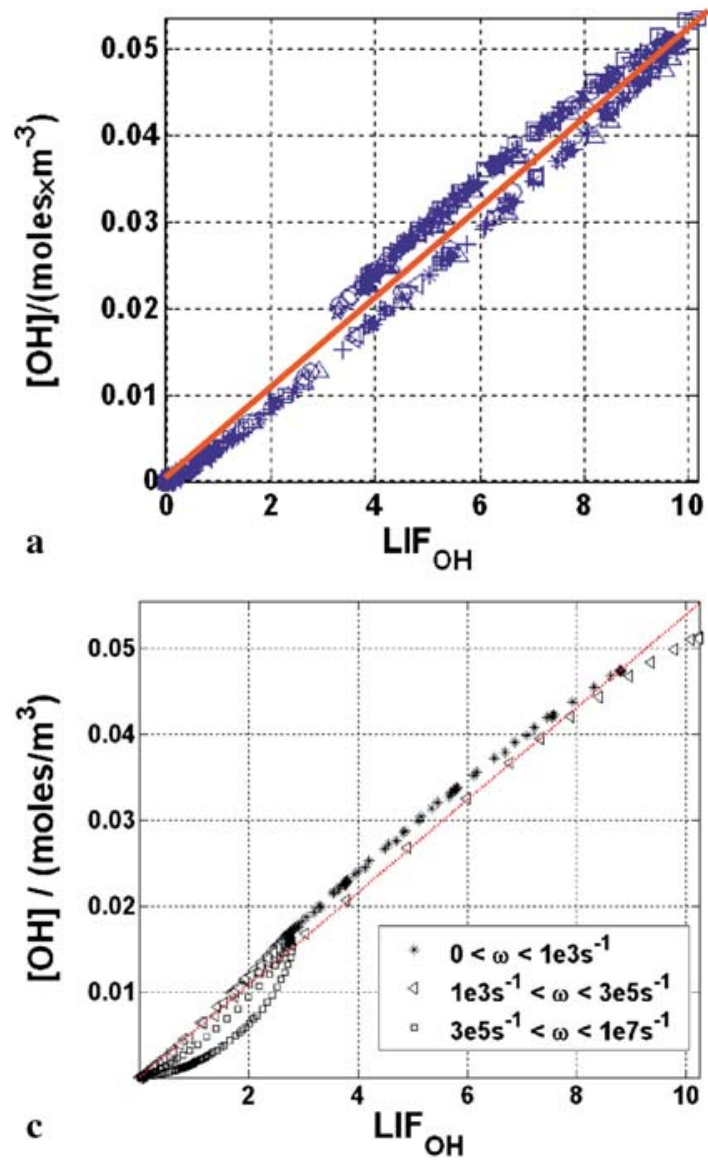
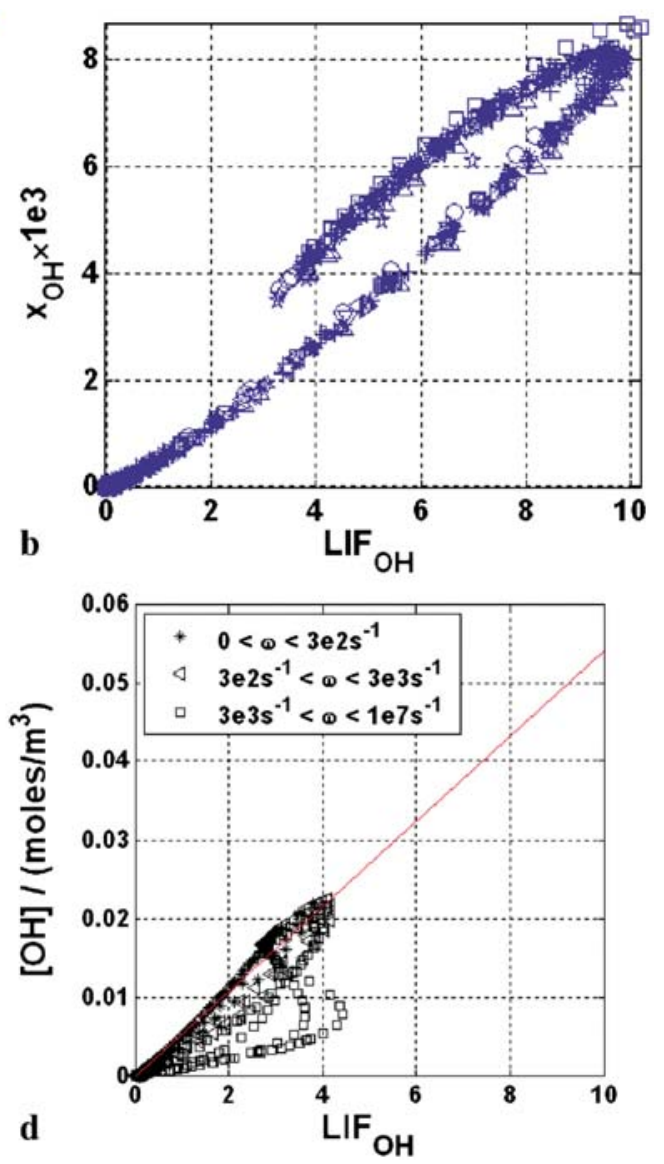

FIGURE 4 a The correlation between $\mathrm{LIF}_{\mathrm{OH}}$ and $[\mathrm{OH}]$ as obtained from 1Dflamelet calculations. The data are collected from calculations for a large range of initial conditions and strain rates ranging from $10 \mathrm{~s}^{-1}$ to $5000 \mathrm{~s}^{-1}$. b Same as in a, but with mole fraction $x_{\mathrm{OH}}$ plotted as a function of $\mathrm{LIFOH}_{\mathrm{OH}}$. c Correlation between $[\mathrm{OH}]$ and $\mathrm{LIF}_{\mathrm{OH}}$, obtained from mixing reactor calculations with different mixing rates $0<\omega<10^{7} \mathrm{~s}^{-1}$ (see legend). The solid line shows the least-squares fit from (a). d Correlation for mixing of air (instead of fuel/air mixture) with the burned gases. Varying mixing rates as indicated in the legend 
In, frame b) the correlation between $\mathrm{LIF}_{\mathrm{OH}}$ and $\mathrm{OH}$ mole fractions $x_{\mathrm{OH}}$ is shown. This correlation is less unique, and, consequently, a measurement of $\mathrm{OH}$ mole fractions $x_{\mathrm{OH}}$ with the method would be less accurate.

For the homogeneous mixing reactor (Fig. $4 \mathrm{c}$ and d), almost exactly the same correlation curve $\mathrm{LIF}_{\mathrm{OH}}-[\mathrm{OH}]$ is obtained as for the flamelet calculations. As is seen in frame 4c), mixing of fresh fuel/air mixture to the burned gas does not affect the correlation, even if extinction occurs (which is the case for $\omega>5 \times 10^{4} \mathrm{~s}^{-1}$; this confirms the findings made with the 1D-flame model. Only in the case of extremely high mixing rates $\left(\omega>3 \times 10^{5} \mathrm{~s}^{-1}\right)$, when the time scale of mixing is in the order of microseconds $\left(10^{-6} \mathrm{~s}\right)$ and less, a slight deviation from the correlation $\mathrm{LIF}_{\mathrm{OH}}-[\mathrm{OH}]$ is observed. This is no limitation to our method in practise, as the mixing time scales in real-world combustion systems are typically in the order of a few $10^{-5} \mathrm{~s} \ldots 10^{-1} \mathrm{~s}$.

To simulate the entrainment of air into the flame, the mixing reactor was studied for the case of air being mixed with burned gas. The result is seen in frame d) of Fig. 4. The effect is similar to the case of high strain rates; for $\omega \ll 3 \times 10^{3} \mathrm{~s}^{-1}$, essentially the same correlation $[\mathrm{OH}]-\mathrm{LIF}_{\mathrm{OH}}$ is obtained as in the case of mixing with fuel/air mixture, albeit with somewhat larger scatter. Again, only for very high mixing speeds a noticeable deviation from the correlation (solid line) is observed. It is interesting to note that in the case of mixing of air, a value of $\mathrm{LIF}_{\mathrm{OH}}>4.2$ is never exceeded by the system.

To summarize the observations, both flamelet and mixing reactor calculations show a remarkably sharp correlation between the LIF-signal and $\mathrm{OH}$ number densities in the flame under study. This correlation is almost linear throughout the whole range of $[\mathrm{OH}]$, and almost unique, within small relative errors (max. $15 \%$ near the region where $\mathrm{LIF}_{\mathrm{OH}}=4$ ).

The sharp correlation between $[\mathrm{OH}]$ and $\mathrm{LIF}_{\mathrm{OH}}$ may appear stunning at a first glance. However, it is merely a manifestation of the fact that, despite quite complex turbulencechemistry interactions being present in the flame, the state space variables $\left(T, p, x_{i}\right)$ are always confined to a lowerdimensional subset of the state space. This behavior is the basis of mechanism reduction methods like the ILDM- (Intrinsic Low-Dimensional Manifolds) method [11,12], or the Computational Singular Perturbation method (CSP [13]). Ultimately, all these methods (as well as ours) exploit the fact that the strongly varying time scales of different chemical reactions and physical processes (molecular transport like diffusion or heat conduction) induce algebraic relations between the concentrations of different species.

From all numerical results, the expression

$$
[\mathrm{OH}]=m \mathrm{LIF}_{\mathrm{OH}}, m=0.00541 \mathrm{~mol} / \mathrm{m}^{3},
$$

is derived as a linear least-squares fit to the data shown in Fig. 4. This expression describes the calculated data points for realistic strain rates $a$ resp. mixing rates $\omega$ to within a relative error in $[\mathrm{OH}]$ of $\pm 15 \%$. Errors would be larger for the case when air is entrained into the flame at a very high mixing rate. In that case, measurement of a second scalar could help to obtain better accuracy. However, in all OH-LIF images we have analyzed, the $\mathrm{LIF}_{\mathrm{OH}}$ profile taken perpendicular to the local flame front-orientation clearly showed values $\mathrm{LIF}_{\mathrm{OH}}$ ris-
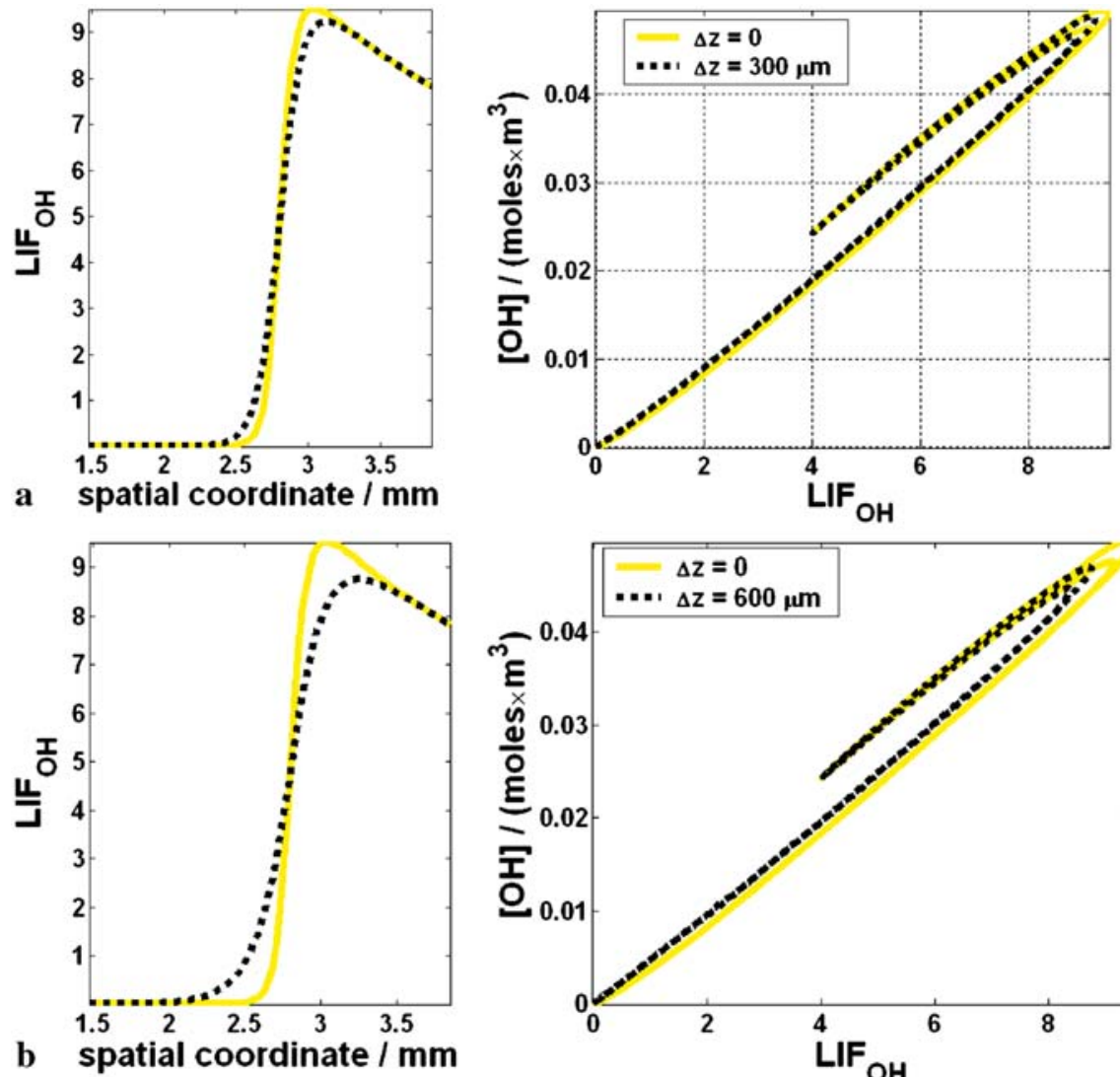

FIGURE 5 a Left: simulated spatial LIF ${ }_{\mathrm{OH}}$ profiles along a flame front, at different spatial resolutions $\Delta z$ : full resolution, and $\Delta z=300 \mu \mathrm{m}$. Bottom: The correlation $[\mathrm{OH}]-\mathrm{LIF}_{\mathrm{OH}}$ for these two resolutions. b same for $\Delta z=600 \mu \mathrm{m}$ 
ing from 0 (in the unburned region) to at least 6 (at the flame front). Therefore, mixing with air can not have occurred to a significant extent in our measurements.

In practical experiments, the available spatial resolution in a measurement is always an issue. [OH] typically increases steeply in flame fronts (at a spatial scale of a few $100 \mu \mathrm{m}$ ), but $\mathrm{LIF}_{\mathrm{OH}}$ (a measured quantity) can change only at a scale that is in the order of magnitude of the experimental spatial resolution. This could affect the correlation $[\mathrm{OH}]-\mathrm{LIF}_{\mathrm{OH}}$, and effectively make it dependent on the spatial resolution. Figure 5 shows spatial profiles of $\mathrm{LIF}_{\mathrm{OH}}$ that are obtained for three different spatial resolutions $\Delta z$ : Full resolution (i.e., an arbitrary small spatial scale can be resolved in the measurement, $\Delta z=0$ ), $\Delta z=300 \mu \mathrm{m}$ (which is close to the spatial resolution of our experiment), and $\Delta z=600 \mu \mathrm{m}$. It is seen that the spatial resolution does not affect the correlation between $\mathrm{LIF}_{\mathrm{OH}}$ and $[\mathrm{OH}]$, if spatially averaged values of $\mathrm{LIF}_{\mathrm{OH}}$ and $[\mathrm{OH}]$ are considered. Note that due to to the limited spatial resolution of an experiment, the measured value of $[\mathrm{OH}]$ is always a spatially averaged version of the real value [OH]. The correlation $\mathrm{LIF}_{\mathrm{OH}^{-}}$ $[\mathrm{OH}]$ remains valid if the spatially averaged value of $[\mathrm{OH}]$ is considered.
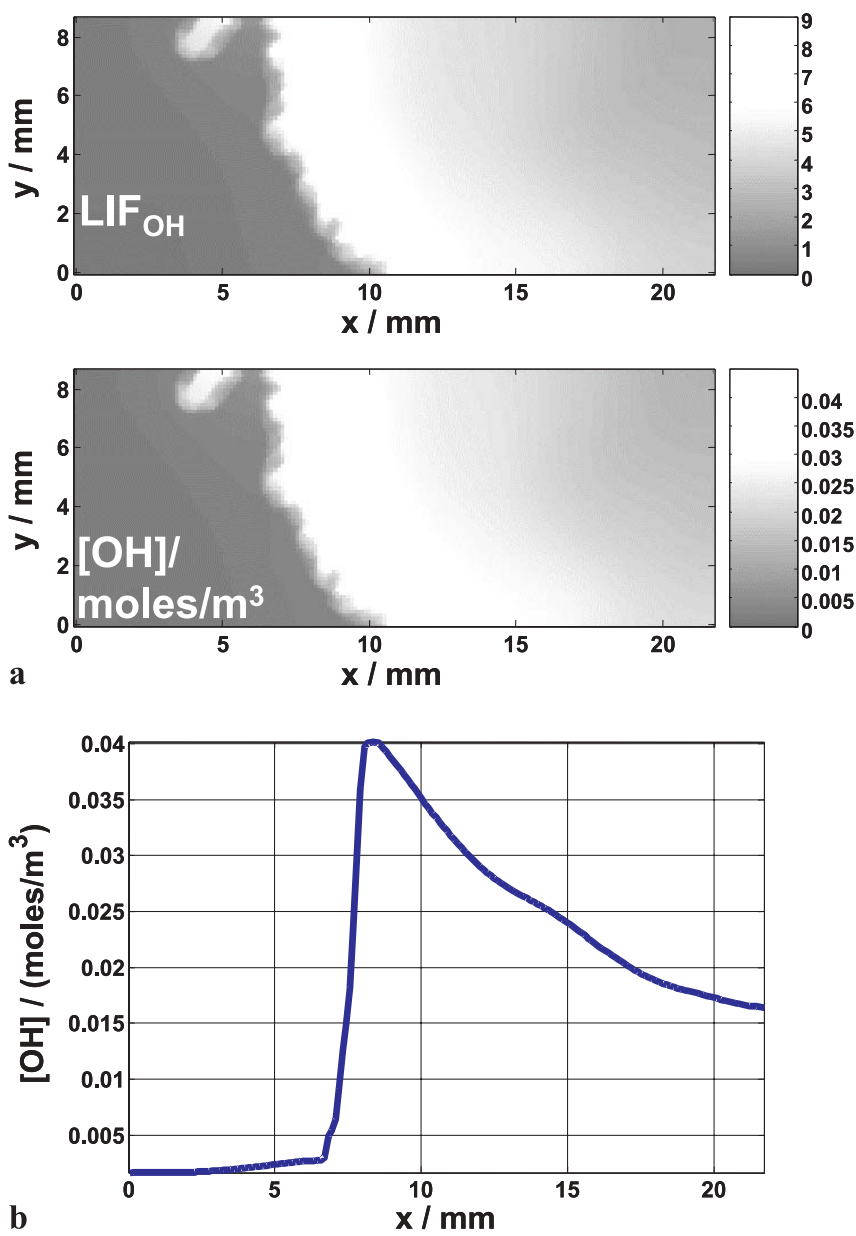

FIGURE 6 a Images of $\mathrm{LIF}_{\mathrm{OH}}$ and $[\mathrm{OH}]$, obtained in a turbulent premixed flame. The corresponding gray-scales are shown to the right of each image. To remove noise from the $\mathrm{LIF}_{\mathrm{OH}}$-image, a nonlinear diffusion filtering technique was applied (see text). b The spatial profile $[\mathrm{OH}]$ along $y=4 \mathrm{~mm}$ in a

\section{2}

\section{Determination of $\mathrm{OH}$ concentrations}

The transformation of measured LIF signals to absolute $\mathrm{OH}$ concentrations is straightforward, using (5). A first application example of this method is displayed in Fig. 6. A measured false-color image of $\mathrm{LIF}_{\mathrm{OH}}$ is shown in a); to remove noise from this image, a nonlinear diffusion technique was applied [14]. Also, the corresponding map of $[\mathrm{OH}]$ is depicted. For our excitation- and detection-scheme, it is the result of the very simple transformation of $\mathrm{LIF}_{\mathrm{OH}}$ values into $\mathrm{OH}$ concentrations (according to (5)).

For all recorded LIF-images, any spatial profile of $\mathrm{LIF}_{\mathrm{OH}}$ taken perpendicular to the flame front displayed values of $\mathrm{LIF}_{\mathrm{OH}}$ clearly exceeding six in regions close to the flame front; we therefore conclude that considerable entrainment of air did not occur at the measured locations (compare Fig. 4d) and the $\mathrm{LIF}_{\mathrm{OH}}$ image in Fig. 6a), so that the correlation $\mathrm{LIF}_{\mathrm{OH}}-[\mathrm{OH}]$ (5) can be applied. The relative error in $[\mathrm{OH}]$ associated with the slight non-uniqueness of the correlation is then below $15 \%$.

These are first results of a preliminary application of this method in an experimental approach. Further measurements will address the application in a wider range of different flame types.

\section{Conclusions}

We have used a combined experimental and numerical approach to measure absolute concentrations of $\mathrm{OH}$ in a turbulent, premixed flame of semi-technical scale. The measurement principle exploits the existence of reduced state spaces: as a consequence of this existence, LIF signals for a particular excitation-detection scheme are strongly correlated to $\mathrm{OH}$ concentrations. This correlation is assessed by a set of detailed numerical simulations of one-dimensional flames and a homogeneous mixing reactor, which were performed for a wide range of conditions and for different models in order to treat molecular transport. It is shown that the correlation for the employed excitation/detection scheme is quite sharp and universal, in a wide range regardless of the initial conditions (temperature of the unburned fuel/air mixture, stoichiometry) and the transport model employed used to describe heat conduction and species diffusion. Especially, non-unity Lewis-number effects or differential diffusion did not affect the correlation, compared to a simple $L e=1$ model. Also, slight changes in the element composition of the gas mixture (due to entrainment of air into the flame gases) or heat losses do not notably affect the correlation. Finally, we investigated the influence of finite spatial resolution in the measurement of LIF signals; it was found that the available spatial resolution in our measurement does not affect the correlation between LIF signal and $[\mathrm{OH}]$.

The ease with which OH-LIF images are transformed into absolute quantitative concentration maps shows how powerful an appropriate combination of numerical simulations and experiments can be. Accuracy and precision of measurements can be enhanced significantly by exploiting correlations between state variables.

An application of the method is demonstrated by a planar measurement of $[\mathrm{OH}]$ in a premixed, turbulent natural-gas/air 
flame. We plan to apply the method to multi-dimensional measurements and to the determination of absolute threedimensional gradients; furthermore, it will be checked if the method can be applied to non-premixed flames.

ACKNOWLEDGEMENTS Financial support by the Deutsche Forschungsgemeinschaft (DFG) within WO175-34 and MA1205/6-2 is gratefully acknowledged.

\section{REFERENCES}

1 E.W. Kaiser, K. Marko, D. Klick, L. Rimai, C.C. Wang, B. Shirinzadeh, D. Zhou: Combust. Sci. Technol. 50, 163 (1986)

2 R. Bender, B. Steiner, D. Schmidt, R. Schieß1, U. Maas: Experimentelle Untersuchung und PDF-simulation turbulenter Vormischflammen, 16. TECFLAM-Seminar, Karlsruhe (2002)
3 R.J. Cattolica, S.R. Vosen: Proc. Comb. Symp. 20, 1273 (1985)

4 J.M. Seitzman, A. Ungut, P.H. Paul, R.K. Hanson: Proc. Comb. Symp. 23, 637 (1991)

5 S. Böckle, J. Kazenwadel, T. Kunzelmann, C. Schulz: Appl. Phys. B 71, $741(2000)$

6 T. Landenfeld, A. Kremer, E.P. Hassel, J. Janicka, T. Schäfer, J. Kazenwadel, C. Schulz, J. Wolfrum: Proc. Combust. Inst. 27, 1023 (1998)

7 W.G. Bessler, C. Schulz, T. Lee, J.B. Jeffries, R.K. Hanson: Appl. Opt. 42, 4922 (2003)

8 Q.V. Nguyen, P.H. Paul: Appl. Phys. B 72, 479 (2001)

9 U. Maas, J. Warnatz: Combust. Flame 74, 53 (1988)

10 G. Stahl, J. Warnatz: Combust. Flame 85, 285, (1991)

11 U. Maas, S.B. Pope: Combust. Flame 88, 239 (1992)

12 A.S. Tomlin, T. Turanyi, M.J. Pilling: In Low Temperature Combustion and Autoignition, ed. by M.J. Pilling (Elsevier, Amsterdam (1997) p. 293

13 S.H. Lam: Combust. Sci. Technol. 89, 375 (1993)

14 J. Weickert: Computing Suppl. 11, 221 (1996) 